\title{
Biological Membranes
}

A Molecular Perspective

from Computation and Experiment 


\section{Biological Membranes}

A Molecular Perspective

from Computation and Experiment

Kenneth M. Merz, Jr. and Benoît Roux Editors

Birkhäuser

Boston • Basel • Berlin 
Kenneth M. Merz, Jr.

Department of Chemistry

Pennsylvania State University

152 Davey Laboratory

University Park, PA 16802

USA
Benoît Roux

Groupe de Recherche en Transport

Membranaire (GRTM)

Départements de physique et de chimie Université de Montréal

C.P. 6128 , succ. Centre-Ville

Montréal, Québec

Canada $\mathrm{H} 3 \mathrm{C} 3 \mathrm{~J} 7$

\section{Library of Congress Cataloging-In-Publication Data}

Biological membranes : a molecular perspective from computation and experiment / Kenneth M. Merz, Jr., and Benoît Roux, editors.

p. $\mathrm{cm}$.

Includes bibliographical references and index.

ISBN 978-1-4684-8582-0 ISBN 978-1-4684-8580-6 (eBook)

DOI 10.1007/978-1-4684-8580-6

1. Membranes (Biology) 2. Lipid membranes. 3. Membrane lipids.

4. Membrane proteins. I. Merz, Kenneth M., 1959- . II. Roux,

Benoît, 1958-

QH601.B485 1996

574.87'5--dc20

95-51668

CIP

Printed on acid-free paper

(C) 1996 Birkhäuser Boston

Softcover reprint of the hardcover 1st edition 1996

Birkhäuser

Copyright is not claimed for works of U.S. Government employees.

All rights reserved. No part of this publication may be reproduced, stored in a retrieval system, or transmitted, in any form or by any means, electronic, mechanical, photocopying, recording, or otherwise, without prior permission of the copyright owner.

Permission to photocopy for internal or personal use of specific clients is granted by Birkhäuser Boston for libraries and other users registered with the Copyright Clearance Center (CCC), provided that the base fee of $\$ 6.00$ per copy, plus $\$ 0.20$ per page is paid directly to CCC, 222 Rosewood Drive, Danvers, MA 01923, U.S.A. Special requests should be addressed directly to Birkhäuser Boston, 675 Massachusetts Avenue, Cambridge, MA 02139, U.S.A.

Cover design by David Gardner, Dorchester, MA.

Typeset by University Graphics, York, PA. 


\section{Contents}

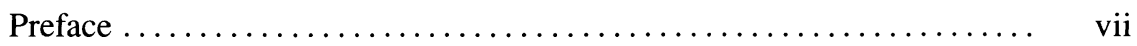

List of Contributors $\ldots \ldots \ldots \ldots \ldots \ldots \ldots \ldots \ldots \ldots \ldots \ldots \ldots \ldots \ldots \ldots \ldots$

Part I: Computational Issues Regarding Biomembrane Simulation

1. Time Scales of Lipid Dynamics and Molecular Dynamics Richard W. Pastor and Scott E. Feller................ 3

2. An Empirical Potential Energy Function for Phospholipids:

Criteria for Parameter Optimization and Applications

Michael Schlenkrich, Jürgen Brickmann,

Alexander D. MacKerell, Jr., and Martin Karplus.........

3. Statistical Mechanics and Monte Carlo Studies of Lipid Membranes

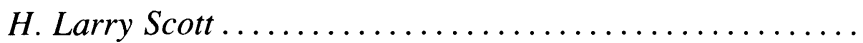

4. Strategic Issues in Molecular Dynamics Simulations of Membranes

Eric Jakobsson, Shankar Subramaniam, and H. Larry Scott

Part II: Experimental Probes of Biomembrane Structure and Dynamics

5. The Liquid-Crystallographic Structure of Fluid Lipid Bilayer Membranes

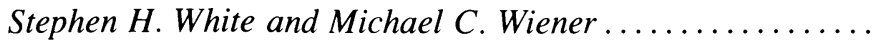

6. Infrared Spectroscopic Determination of Conformational Disorder and Microphase Separation in Phospholipid Acyl Chains

Richard Mendelsohn and Robert G. Snyder. 
7. Membrane Structure and Dynamics Studied with

NMR Spectroscopy

Michael F. Brown

Part III: Small Molecules and Peptides in Biomembranes

8. Movement of Small Molecules in Lipid Bilayers:

Molecular Dynamics Simulation Studies

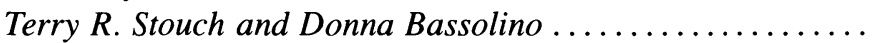

9. Structural Basis and Energetics of Peptide Membrane Interactions Huey W. Huang ..............................

10. Computational Refinement Through Solid State NMR and Energy Constraints of a Membrane Bound Polypeptide

Randal R. Ketchem, Benoît Roux, and Timothy A. Cross.....

11. Bilayer-Peptide Interactions

K. V. Damodaran and Kenneth M. Merz, Jr...........

\section{Part IV: Membrane Proteins}

12. Peripheral Membrane Proteins

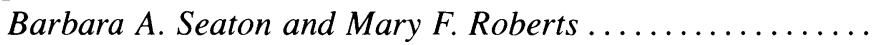

13. Thermodynamics of the Interaction of Proteins with Lipid Membranes

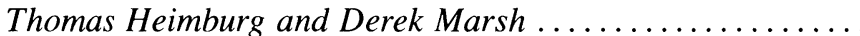

14. Role of Lipid Organization and Dynamics for Membrane Functionality

Ole G. Mouritsen and Paavo K. J. Kinnunen ...

15. Prediction of the Structure of an Integral Membrane Protein:

The Light-Harvesting Complex II of Rhodospirillum molischianum

Xiche Hu, Dong Xu, Kenneth Hamer, Klaus Schulten,

Juergen Koepke, and Hartmut Michel.

16. Monte Carlo Models of Spontaneous Insertion of Peptides into Lipid Membranes

Jeffrey Skolnick and Mariusz Milik

17. Molecular Dynamics of Pf1 Coat Protein in a

Phospholipid Bilayer

Benoît Roux and Thomas B. Woolf

Index 


\section{Preface}

The interface between a living cell and the surrounding world plays a critical role in numerous complex biological processes. Sperm/egg fusion, virus/cell fusion, exocytosis, endocytosis, and ion permeation are a few examples of processes involving membranes. In recent years, powerful tools such as X-ray crystallography, electron microscopy, nuclear magnetic resonance, and infra-red and Raman spectroscopy have been developed to characterize the structure and dynamics of biomembranes. Despite this progress, many of the factors responsible for the function of biomembranes are still not well understood. The membrane is a very complicated supramolecular liquid-crystalline structure that is largely composed of lipids, forming a bilayer, to which proteins and other biomolecules are anchored. Often, the lipid bilayer environment is pictured as a hydrophobic structureless slab providing a thermodynamic driving force to partition the amino acids of a membrane protein according to their solubility. However, much of the molecular complexity of the phospholipid bilayer environment is ignored in such a simplified view. It is likely that the atomic details of the polar headgroup region and the transition from the bulk water to the hydrophobic core of the membrane are important. An understanding of the factors responsible for the function of biomembranes thus requires a better characterization at the molecular level of how proteins interact with lipid molecules, of how lipids affect protein structure and of how lipid molecules might regulate protein function. Computer simulations of detailed atomic models based on realistic microscopic interactions represent a powerful approach to gain insight into the structure and dynamics of complex macromolecular systems such as a biomembrane. At the present time, even qualitative information gained from such computer simulations is valuable. Nevertheless, extension of current computational methodologies to simulate biomembrane systems still represents a major challenge. However, this field is just in its infancy, and it is likely that both experimental and theoretical tools will be needed to solve these problems. It is the goal of the present volume to provide a concise overview of computational and experimental advances in the understanding of lipid bilayers and protein/lipid interactions at the molecular level. 
It can be reasonably expected that molecular simulations will play an increasingly important role in the future. While most trajectories to date are confined to the $1 \mathrm{~ns}$ time regime, this clearly will not be the case in the coming years. Just what kinds of time scales will we be able to simulate in the next five years? As an illustration, let us consider the following analysis. A typical simulation for a biological system now consists of $\sim 15,000$ atoms, which on modern parallel and vector supercomputers requires approximately 1 hour to generate 1 ps of a trajectory. Thus, one individual running MD simulations continuously can generate at most $\sim 9$ ns of trajectories in one year utilizing modern vector and parallel hardware resources available at many universities and supercomputer centers. Furthermore, it can be expected that algorithmic capabilities will be enhanced by a factor of 2 to 10 -fold in the next year. This suggests that in one year we will go from a 9 ns capability to a 18 ns-90 ns capability just by improving our computational algorithms. In addition, it is generally agreed that there is a twofold speed increase every 18 months in computer technology. Thus, by the end of five years we can estimate a tenfold increase in computer power alone. Hence, the 9 ns/year we estimate now will increase to $90 \mathrm{~ns}$ based only on an increase in computer performance. Including an algorithmic improvement of 2 to 10 -fold on top of this leads to an estimate of a $180 \mathrm{~ns}$ to 0.9 micros/year capability for one individual only. Obviously, this analysis is just an estimate and neglects many factors (e.g., increased system sizes, increased potential function complexity, etc.), but we think it is clear that molecular simulations on phospholipid bilayers will reach near microsecond capabilities in the next five years.

It is clear that theoretical methods will evolve to the point where we can address very long time scale issues. How about experimental techniques? Clearly, new approaches to solve experimental problems involving biomembranes will be developed in the coming years. Furthermore, we can also expect significant improvements in the techniques used to study biomembranes. For example, NMR has enjoyed tremendous growth over the years and this will continue in the coming years. The field strength of magnets have continued to grow, which provides higher resolution information that can be used to analyze biomembrane structure and dynamics. Moreover, new Solid State NMR techniques for oriented samples continue to be developed that will improve the ability to analyze biological membrane systems. Similarly, other experimental techniques like neutron scattering, X-ray, IR, CD, etc., will also continue to be improved upon in the coming years. Hence, the combination of improved computational and experimental techniques indicate that there is a bright future for the continued investigation of the structure, function, and dynamics of biological membranes at the molecular level.

This volume is separated into four sections. In section I, the basic theoretical and computational issues regarding biomembrane structure and dynamics are addressed. These issues range from basic statistical mechanics to force field development and evaluation. Thus, this section contains the necessary information required for anyone interested in attempting to model biomembranes using 
molecular dynamics or Monte Carlo methods. Section II then moves onto a series of chapters describing experimental probes that can be used to assess biomembrane structure. These include X-ray, IR and NMR techniques, and all are capable of providing microscopic or macroscopic insights that can be used to enhance our understanding of biomembrane structure and dynamics. Moreover, these experimental techniques generate information that can be used to assess and verify theoretical studies. Section III gives both a theoretical and experimental perspective on the interaction of peptides with biomembranes. Many peptides are membrane active and deserve study in their own right, but these systems can also serve as powerful models of protein/lipid interactions. Hence, by understanding these smaller (and hopefully less complicated) systems we will increase our understanding of the larger integral membrane class of proteins. Finally, Section IV gives a broad theoretical and experimental perspective of protein/lipid interactions. In this section the chapters give insights into the thermodynamics of protein lipid interactions as well as provide structural details of these systems.

The editors would like to thank the staff at Birkhäuser and the contributors for helping us produce an outstanding volume on recent advances towards understanding the structure, function and dynamics of biomembranes and lipid/protein interactions. 


\section{List of Contributors}

Donna Bassolino, Bristol-Myers Squibb Pharmaceutical Research Institute, P.O. Box 4000, Princeton, NJ 08543-4000, USA

Jürgen Brickmann, Institut für Physikalische Chemie, Technische Hochschule Darmstadt, Petersenstraße 20, D-6100 Darmstadt, Federal Republic of Germany

Michael F. Brown, Department of Chemistry, University of Arizona, Tucson, AZ 85721, USA

Timothy A. Cross, Center for Interdisciplinary Magnetic Resonance at the $\mathrm{Na}$ tional High Magnetic Field Laboratory, Institute of Molecular Biophysics and Department of Chemistry, Florida State University, 1800 E. Paul Dirac Dr., Tallahassee, Fl 32306-3016, USA

K.V. Damodaran, Department of Chemistry, Pennsylvania State University, 152 Davey Laboratory, University Park, PA 16802, USA

Scott E. Feller, Biophysics Laboratory, Center for Biologics Evaluation and Research, Food and Drug Administration, 1401 Rockville Pike, Rockville, MD, 20852-1448, USA

Kenneth Hamer, Theoretical Biophysics, Beckman Institute, University of Illinois at Urbana-Champaign, Urbana, IL 61801, USA

Thomas Heimburg, Abteilung Spektroskopie, Max-Planck-Institute für Biophysikalische Chemie, Postfach 2841, D-37018 Göttingen, Germany

Xiche Hu, Theoretical Biophysics, Beckman Institute, University of Illinois at Urbana-Champaign, Urbana, IL 61801, USA

Huey W. Huang, Department of Physics, Rice University, 6100 Main Street, Houston, TX 77005-1892, USA 
Eric Jakobsson, National Center for Supercomputing Applications, 4039 Beckman Institute, University of Illinois, 405 North Mathews Ave, Urbana, IL 61801, USA

Martin Karplus, Department of Chemistry, Harvard University, 12 Oxford St, Cambridge, MA 02138, USA; and Laboratoire de Chimie Biophysique, Institut le Bel, Université Louis Pasteur, 67000 Strasbourg, France

Randal R. Ketchem, Center for Interdisciplinary Magnetic Resonance at the National High Magnetic Field Laboratory, Florida State University, 1800 E. Paul Dirac Dr., Tallahassee, Fl 32306-3016, USA

Paavo K.J. Kinnunen, Department of Medical Chemistry, Institute of Biomedicine, POB 8, FIN-00014, University of Helsinki, Finland

Juergen Koepke, Max-Plank-Institut für Biochemie, Abteilung Molekulare Membranbiologie, 6000 Frankfurt, Germany

Alexander D. MacKerell, Jr., Department of Pharmaceutical Sciences, School of Pharmacy, University of Maryland at Baltimore, 20 North Pine Street, Baltimore, MD 21201-1180, USA; and Department of Chemistry, Harvard University, 12 Oxford Street, Cambridge, MA 02138, USA

Derek Marsh, Abteilung Spektroskopie, Max-Planck-Institute für Biophysikalische Chemie, Postfach 2841, D-37018 Göttingen, Germany

Richard Mendelsohn, Department of Chemistry, Newark College, Rutgers University, 73 Warren Street, Newark, NJ 07102, USA

Kenneth M. Merz, Jr., Department of Chemistry, Pennsylvania State University, 152 Davey Laboratory, University Park, PA 16802, USA

Mariusz Milik, Department of Molecular Biology, Scripps Research Institute, 10666 N. Torrey Pines Road, MB1, La Jolla, CA 92037, USA

Hartmut Michel, Max-Plank-Institut für Biochemie, Abteilung Molekulare Membranbiologie, 6000 Frankfurt, Germany

Ole G. Mouritsen, Department of Physical Chemistry, Technical University of Denmark, Bldg 206, DK-2800 Lyngby, Denmark

Richard W. Pastor, Biophysics Laboratory, Center for Biologics Evaluation and Research, Food and Drug Administration, 1401 Rockville Pike, Rockville, MD, 20852-1448, USA

Mary F. Roberts, Merkert Chemistry Center, Boston College, Chestnut Hill, MA 02167, USA

Benoît Roux, Groupe de Recherche en Transport Membranaire (GRTM), Départements de physique et de chimie, Université de Montréal, C.P. 6128, succ. Centre-Ville, Montréal, Québec, Canada H3C 3J7 
Michael Schlenkrich, Institut für Physikalische Chemie, Technische Hochschule Darmstadt, Petersenstraße 20, D-6100 Darmstadt, Federal Republic of Germany; and Silicon Graphics, Erlenstraesschen 65, CH-4125 Riehen, Switzerland; and Department of Chemistry, Harvard University, 12 Oxford St, Cambridge, MA 02138, USA

Klaus Schulten, Theoretical Biophysics, Beckman Institute, University of Illinois at Urbana-Champaign, Urbana, IL 61801, USA

H. Larry Scott, Department of Physics, Oklahoma State University, Stillwater, OK 74078-3072, USA

Barbara A. Seaton, Structural Biology Group, Department of Physiology, Boston University School of Medicine, 80 E. Concord St., Boston, MA 02118, USA

Jeffrey Skolnick, Department of Molecular Biology, Scripps Research Institute, 10666 N. Torrey Pines Road, MB1, La Jolla, CA 92037, USA

Robert G. Snyder, Department of Chemistry, University of California, Berkeley, CA 94720-1460, USA

Terry R. Stouch, Bristol-Myers Squibb Pharmaceutical Research Institute, Room H3812, P.O. Box 4000, Princeton, NJ 08543-4000, USA

Shankar Subramaniam, National Center for Supercomputing Applications, 4041 Beckman Institute, University of Illinois, 405 North Mathews Ave, Urbana, IL 61801, USA

Stephen H. White, Department of Physiology and Biophysics, School of Medicine, University of California at Irvine, Irvine, CA 92717-4560, USA

Michael C. Wiener, Department of Biochemistry and Biophysics, University of California at San Francisco, San Francisco, CA 94143-0448, USA

Thomas B. Woolf, Department of Physiology, Johns Hopkins University School of Medicine, 725 N. Wolfe Street, Baltimore, MD 21205, USA

Dong Xu, Theoretical Biophysics, Beckman Institute, University of Illinois at Urbana-Champaign, Urbana, IL 61801, USA 